\title{
Microsurgical unilateral laminotomy for bilateral decompression of degenerative lumbar canal stenosis: a comparative study
}

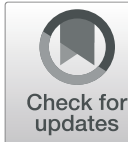

\author{
Mohamed I. Refaat ${ }^{*}$ (D), Amr K. Elsamman', Adham Rabea² and Mohamed I. A. Hewaidy²
}

\begin{abstract}
Background: The quest for better patient outcomes is driving to the development of minimally invasive spine surgical techniques. There are several evidences on the use of microsurgical decompression surgery for degenerative lumbar spine stenosis; however, few of these studies compared their outcomes with the traditional laminectomy technique.
\end{abstract}

Objectives: The aim of our study was to compare outcomes following microsurgical decompression via unilateral laminotomy for bilateral decompression (ULBD) of the spinal canal to the standard open laminectomy for cases with lumbar spinal stenosis.

Subjects and methods: Cases were divided in two groups. Group (A) cases were operated by conventional full laminectomy; Group (B) cases were operated by (ULBD) technique. Results from both groups were compared regarding duration of surgery, blood loss, perioperative complication, and postoperative outcome and patient satisfaction.

Results: There was no statistically significant difference between both groups regarding the improvement of visual pain analogue, while improvement of neurogenic claudication outcome score was significant in group (B) than group (A). Seventy-three percent of group (A) cases and $80 \%$ of group (B) stated that surgery met their expectations and were satisfied from the outcome.

Conclusion: Comparing ULBD with traditional laminectomy showed the efficacy of the minimally invasive technique in obtaining good surgical outcome and patient satisfaction. There was no statistically significant difference between both groups regarding the occurrence of complications The ULBD technique was found to respect the posterior spinal integrity and musculature, accompanied with less blood loss, shorter hospital stays, and shorter recovery periods than the open laminectomy technique.

Keywords: Lumbar spinal stenosis, Unilateral laminotomy, Laminectomy, Neurogenic claudication, Quality of life

\section{Introduction}

Degenerative lumbar spinal stenosis (LSS) is a progressive degenerative disease, more commonly to occur over age of 60 years. Causes of degenerative LSS include broad base disc bulging, ligamentum flavum hypertrophy, and degenerative facet joint disease. It significantly impacts the quality of life in performing the daily activities and can lead to progressive disability [1]. Patients usually present clinically

\footnotetext{
* Correspondence: mrefaat_77@yahoo.co.uk

'Department of Neurosurgery, School of Medicine, Cairo University, Elmanial, Cairo, Egypt

Full list of author information is available at the end of the article
}

with chronic low back pain followed by neurogenic claudication pains, which is exacerbated by standing and walking, relieved by rest, leaning forwards, and flexion of the waist (typically in seated position). Patients may later on experience tingling, numbness, and progressive lower limb weakness [2].

The primary treatment line for most patients with lumbar spinal stenosis is non-surgical [3]. Degenerative LSS is a chronic condition, and in many patients, nonsurgical management fails to achieve satisfactory relief of symptoms. The study published by Phan and colleagues found that in a conservatively managed cohort $15 \%$ only 
experienced relief of symptoms after 4 years, $70 \%$ described stationary course, and 15\% reported worsened symptoms [4].

Surgery is indicated for chronic cases who fail to respond to conservative management or for acute cases presenting with progressive neurologic dysfunction. The traditional management for lumbar canal stenosis was the formal laminectomy with/without medial facetectomy $[5,6]$.

Minimally invasive surgical techniques are recently gaining popularity; they are less traumatic to the back muscles and preserve the integrity of the midline bony structures and the interspinous ligaments. These techniques include (1) hemi laminotomy decompression, (2) interlaminar decompression, (3) interspinous spacers, and (4) spinous process-splitting laminectomy [7].

The objective of this study was to evaluate the efficacy and safety of microscopic unilateral laminotomy for bilateral decompression (ULBD) of lumbar spinal stenosis and to compare the outcome with conventional full laminectomy.

\section{Subjects and methods}

This is a retrospective study conducted on data of 60 patients, who were indicated for surgical decompression for lumbar spinal stenosis. Cases were managed in the period between January 2017 and January 2018.

Lumbar spinal stenosis was diagnosed clinically by presence of low back pain, neurogenic claudication pains, and/or radiculopathies. Diagnosis was confirmed by the presence of bony, ligamentous, or discogenic canal stenosis in lumbar spine MRI. All cases had an initial period of conservative therapies for at least 3 months. Cases with spinal deformities and instabilities, as well as recurrent cases, were excluded from the study group.

Cases included in this study were allocated in two groups. Group (A) cases were operated by bilateral full laminectomy and medial facetectomies. In group (B) cases, unilateral laminotomy and bilateral decompression (ULBD) was performed.

In group (A) cases, bilateral full laminectomy was performed. The technique was performed under general anesthesia. Subperiosteal muscle separation was performed bilaterally till reaching the desired laminae. Full laminectomy was then performed, including the ligamentum flavum above and below the excised lamina. Finally bilateral medial facetectomy, foraminotomy, and inspecting the intervertebral discs were performed. ULBD technique was performed under general anesthesia, in prone position. C-arm fluoroscopy was utilized to identify the desired level before skin incision. Subperiosteal muscle separation followed till reaching the desired lamina. Ipsilateral microscopic laminotomy is then performed, followed by excision of the ligamentum flavum and trimming of the medial aspect of the facet joint, decompressing the ipsilateral foramen and inspecting for any disc fragments. The operating table and the surgical microscope were then tilted to the opposite side followed by the removal of the base of the spinous process to allow direct vision to the contralateral side. We then undermined the opposite lamina, removed the ligamentum flavum, and did the facetal trimming and the foraminotomy directly from the opposite side.

Results from both groups were compared regarding duration of surgery, blood loss, perioperative complication, and postoperative outcome and patient satisfaction using visual analogue scale (VAS) and neurogenic claudication outcome score (NCOS) [8]. Analysis of data was performed using IBM SPSS v. 22 for Windows (2016, IBM, USA)

\section{Results}

This study included sixty cases with lumbar spinal stenosis, divided into two equal groups. Group (A) cases were operated upon by bilateral full laminectomy, while group (B) cases were operated upon by microscopic unilateral laminotomy and bilateral decompression. In group (A), the mean age of cases was 57.2 years (8.8 SD), and the male to female ratio was 53:47. In group (B) cases, the mean age was 59.4 (9.2 SD), and the male to female ratio was 60:40. Multivariate analysis of age and postoperative improvement of the NCOS showed no statistically significant effect of the mean age on the postoperative NCOS $(p>0.05)$. The most common presenting symptom was low back pain and claudication pains in both groups, followed by sciatic pain. Motor affection was present in $26.6 \%$ of group (A) cases and in $20 \%$ of group (B) cases. The most common affected level was L4/5 (63\%), followed by L3/4 (20\%) and L5S1 (17\%) in both groups. The mean duration of symptoms till the operation was 27.2 months and 19.1 months (SD 14.5, 13.4) for group A and group B, respectively. No statistical significance was detected in any of these demographic data in both groups.

Thirty six patients $(60 \%)$ were decompressed at one level (16 in group A and 20 in group B), 16 patients (26.6\%) were decompressed at two levels (9 in group A and 7 in group B), and 8 patients (13.3\%) were decompressed at three levels ( 5 in group A and 3 in group B). Total number of decompressed levels was 9; the most affected level was L4-5 (63\%) followed by L3-4 (20\%) and L5-S1 (17\%) respectively. Fourteen patients (23.3\%) required discectomy.

Discectomy was needed in $26.7 \%$ of group (A) cases (8 cases) and in $20 \%$ of group (B) cases (6 cases). The average blood loss was significantly lower in group (B) cases 
than in group (A), $128.7 \mathrm{ml}$ (SD 35.4) and $174.4 \mathrm{ml}$ (SD 42.9). The mean operative time was lower in group (A) than in group (B); $83 \mathrm{~min}$ (SD 22.9) and 102.8 (16.5). The mean follow-up period was 9.9 months (SD 1.5) and 8.3 months (SD 3.2) in both groups respectively.

The mean postoperative VAS for pain improved by 5.9 points compared with 5.7 in the group A and B respectively which was not statistically significant $(P>0.5)$. While the mean postoperative NCOS improved by 37.6 points compared with 41.1 in the group $\mathrm{A}$ and $\mathrm{B}$ respectively which was statistically significant $(P=0.05)$ (Fig. 1) However the preoperative NCOS for group B was around 10 points more than that of group A, this was statistically significant difference which abolishes the significant difference in postoperative NCOS (Table 1). Seventy-three percent of group (A) cases and $80 \%$ of group (B) stated that surgery met their expectations and were satisfied from the outcome (Fig. 2).

There was no statistically significant difference between both groups regarding the occurrence of complications as there was no complications in 28 cases (93.3\%) in group (A) and 29 (96.6\%) in group (B). There were only 2 cases (6.6\%) complicated with the dural tear group (A) versus 1 case (3.3\%) complicated with dural tear in group (B) $(P$ value $=0.475)$.

\section{Discussion}

Lumbar canal stenosis is a common indication for decompressive spine surgery. Decompression is indicated for cases who fail to respond to medical management or cases with severe clinical symptoms [9].

The target of lumbar spinal stenosis surgery is decompression of the neural canal and the lumbar foramina while preserving much of the anatomy and the biomechanics of the lumbar spine. The traditional surgeries of wide laminectomy, medial facetectomy, and foraminotomy are accused of causing local tissue trauma and postoperative instability, which increases in the need for fusion procedures.

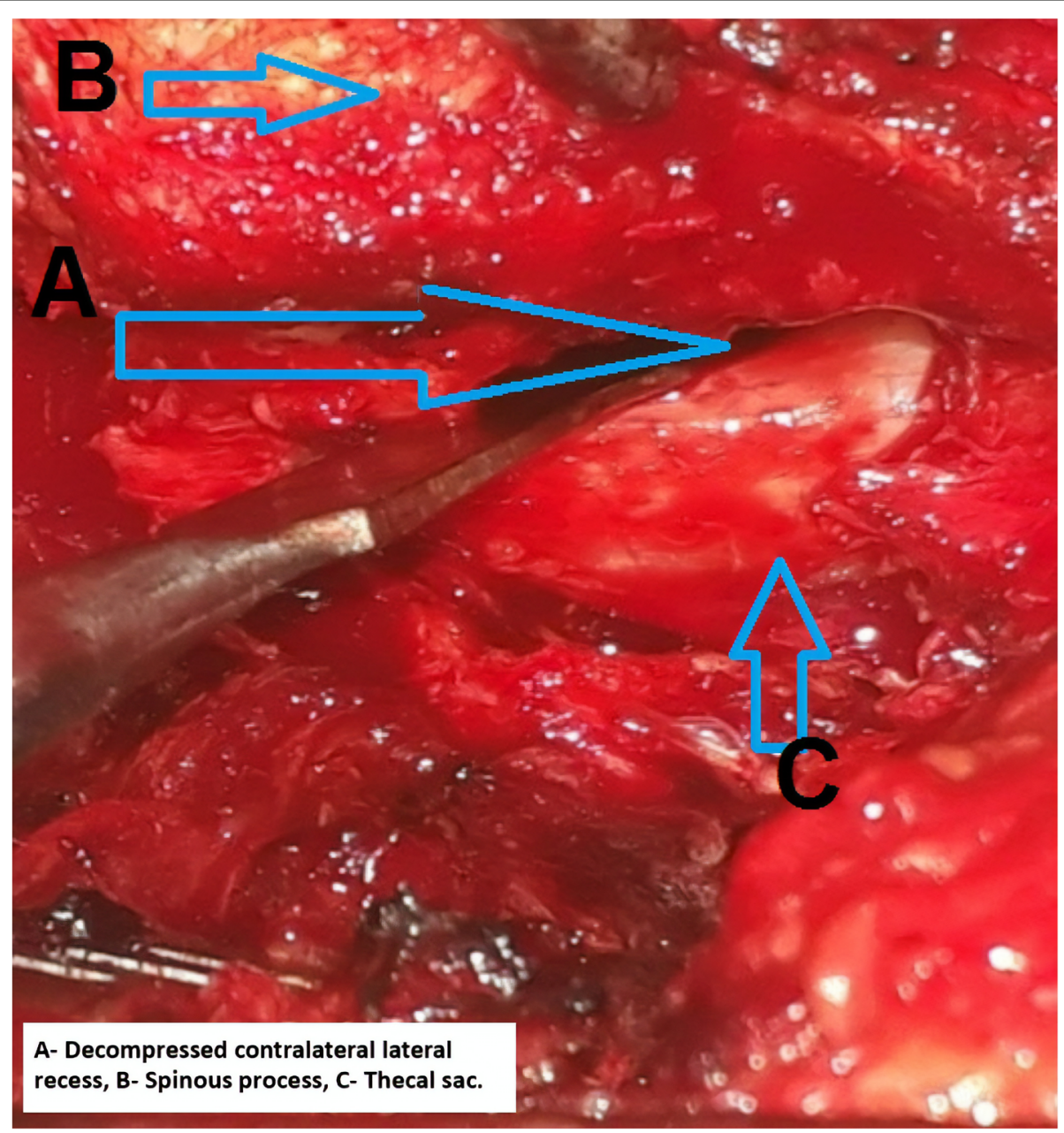

Fig. 1 Intraoperative image showing the unilateral laminotomy and bilateral decompression technique 
Table 1 Summary of patient's data

\begin{tabular}{lllll}
\hline & Group A $(n=30)$ & Group B $(n=30)$ & $p$ value & Statistical significance \\
\hline VAS pre-op (mean) for pain & 7.2 & 7.2 & 0.935 & Non-significant \\
VAS post-op (mean) for pain & 1.3 & 1.5 & 0.653 & Non-significant \\
NCOS pre-op (mean) & 25 & 34.5 & 0.150 & Non-significant \\
NCOS post-op (mean) & 62.6 & 75.6 & 0.775 & Non-significant \\
\hline
\end{tabular}

$n$ Number, VAS Visual analogue scale, NCOS Neurogenic Claudication Outcome Score

Several studies described the ULBD technique; showing good outcomes, less blood loss, and reasonable operation times. Few of these reports compared ULBD technique and open laminectomy, which is still a common practice in our country that is why we conducted this comparative study between both techniques [8].

We performed our laminotomy through the more symptomizing or the radiologically more stenotic side. Proper undercutting of the spinous process, tilting of the operating table, and the use of the surgical microscope allowed adequate visualization of the contralateral lateral recess and foramen. The main advantage of the technique is sparing the midline bony and ligamentous structures, as well as the contralateral lumbar muscles.

Concerning the number of levels operated, in the study published by El Morshidy and colleagues 2016, $36.8 \%$ were decompressed in one level, $39.6 \%$ two levels, $19.8 \%$ three levels, and $3.8 \%$ four levels. The most common level was also L4/5, followed by L3/4 and L5S1. In $16 \%$ of cases, discectomy was needed [10]. This was similar to our studied cases.

The length of operation was significantly higher in group B (102.8 $\mathrm{min} /$ level) than group A ( $83 \mathrm{~min} /$ level); this was like the results published by Khoo and colleagues who reported an operative duration of $109 \mathrm{~min}$ for a single level micro-endoscopic unilateral laminotomy and $88 \mathrm{~min}$ for open laminectomy [11]. In our study, upgrading our skills in microscopic approach for decompression of lumbar canal stenosis and use of hemi-laminectomy retractors and Kerrison rongeur instead of tubular retractors and drill increased the operative time needed for surgery. The mean blood loss was significantly higher among group A than group B which is matching but lesser amount than results described by Thomé and colleagues [12].

The first step in evaluation of a new of a new surgical technique must be analysis of its safety compared with the current standard of care. Our study did not show any significant difference between the complication rates of both groups. All the complications met in the study were dural tears (5\% of cases); we did not meet any root injuries or postoperative increase in radiculopathy. These facts matched the results published by Çavuşoğlu and colleagues, who showed durotomy rates for laminectomy to range from 5 to $15 \%$ and unilateral laminotomy with contralateral decompression in $3.5-12 \%$ [9]. None of our cases showed postoperative instability that required further intervention.

There was no statistically significant difference between both groups regarding the visual pain analogue scale preand postoperative as well as the neurogenic claudication outcome score (NCOS) pre- and postoperative. In the retrospective study conducted by Den Boogert and colleagues, patients in the ULBD group reported better overall

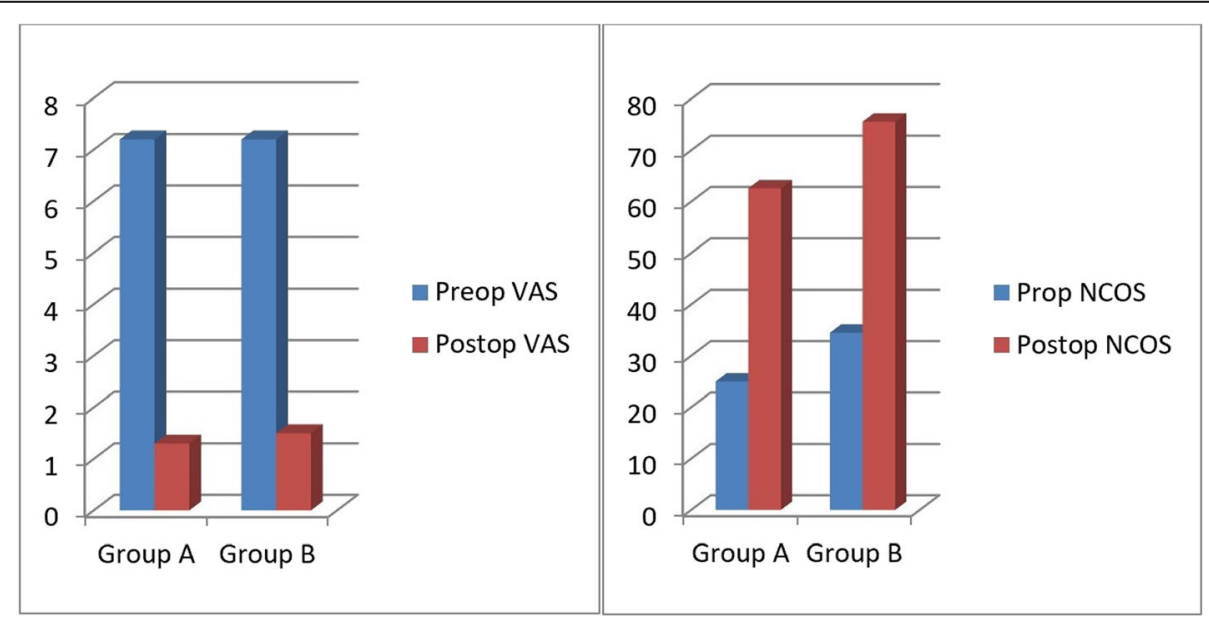

Fig. 2 Summary of pre- and postoperative mean VAS and NCOS 
satisfaction with the procedure and a reduction in visual analogue scale [4].

The small number of cases and the short follow-up period were the main limitations of our study as increasing evidence suggests that outcomes may deteriorate over time [8].

\section{Conclusion}

Minimally invasive surgical techniques for lumbar spinal decompression are gaining more popularity as life expectancy increases and the increased focus on improving the quality of life of the elderly. Standard open wide laminectomy has been used for many years as the standard technique for decompression of the lumbar canal. Comparing microscopic unilateral laminotomy and bilateral decompression with traditional wide laminectomy showed the efficacy of the minimally invasive technique in obtaining good surgical outcome and patient satisfaction. The ULBD technique was found to respect the posterior spinal integrity and musculature, accompanied with less blood loss, shorter postoperative hospital stays, and shorter recovery periods than the open laminectomy technique.

\section{Acknowledgements}

Not applicable.

\section{Authors' contributions}

All authors read and approved the final manuscript. AKE had substantial contribution to the conception, idea, and design of the work. MAH analyzed and interpreted the patient data. MIH and AR have shared in writing the manuscript. All authors shared in doing the surgical intervention for the cases. All authors read and approved the final manuscript.

\section{Funding}

This study was self-funded by the authors.

\section{Availability of data and materials}

The datasets used and/or analyzed during the current study are available from the corresponding author on reasonable request.

\section{Ethics approval and consent to participate}

The research protocol of this study was approved by the "Faculty Council of Kasr Alainy medical school, Cairo University" on 20 February 2017.

All cases included in this study have signed our institutional consent before performing their surgeries including their approval of participation in any future studies.

\section{Consent for publication}

All cases included in this study have signed a consent for approval of publication of their data.

\section{Competing interests}

The authors declare that they have no competing interests.

\section{Author details}

'Department of Neurosurgery, School of Medicine, Cairo University, Elmanial, Cairo, Egypt. ${ }^{2}$ Department of Neurosurgery, School of Medicine, Beni Suef University, Beni Suef, Egypt.
Received: 2 July 2019 Accepted: 29 October 2019

Published online: 28 November 2019

References

1. Katz JN, Harris MB. Lumbar spinal stenosis. N Engl J Med. 2008;358:818-25.

2. Suri P, Rainville J, Kalichman L, Katz JN. Does this older adult with lower extremity pain have the clinical syndrome of lumbar spinal stenosis? JAMA. 2010;304:2628-36.

3. Atlas SJ, Delitto A. Spinal stenosis: surgical versus nonsurgical treatment. Clin Orthop Relat Res. 2006;443:198-207.

4. Phan K, Teng I, Shultz K, Mobbs RJ. Treatment of lumbar spinal stenosis by microscopic unilateral laminectomy for bilateral decompression: a technical note. Orthop Surg. 2017;9:241-6.

5. Weinstein JN, Lurie JD, Tosteson TD, Skinner JS, Hanscom B, Ana T, et al. Surgical vs. Nonoperative treatment for lumbar disk herniation: The Spine Patient Outcomes Research Trial (SPORT) observational cohort. JAMA. 2006; 296:2451-9.

6. Försth P, Ólafsson G, Carlsson T, Frost A, Borgstrom F, Fritzel P, et al. A randomized, controlled trial of fusion surgery for lumbar spinal stenosis. $\mathrm{N}$ Engl J Med. 2016:374:1413-23.

7. Richard A. Lumbar laminectomy: classical versus minimally invasive surgical technique. In: Surgery of the Spine and Spinal Cord. Cham: Springer; 2016. p. 497-512.

8. Mobbs RJ, Sivabalan P, Raley D, Rao P. Outcomes after decompressive laminectomy for lumbar spinal stenosis: comparison between minimally invasive unilateral laminectomy for bilateral decompression and open laminectomy. J Neurosurg Spine. 2014;21:179-86.

9. Çavuşoğlu H, Raya KA, Türkmenoglu ON, Tuncer C, Colak I, Aydin U. Midterm outcome after unilateral approach for bilateral decompression of lumbar spinal stenosis. Eur Spine J. 2007;16:2133-42.

10. El Morshidy EM, Ali HM, Khalifa YE, ELkady HA, Siepe CJ, Mayer MH. Microscopic decompression for lumbar spinal canal stenosis. The Egyptian Orthopaedic Journal. 2016;51:158-64.

11. Khoo LT, Fessler RG. Microendoscopic decompressive laminotomy for the treatment of lumbar stenosis. Neurosurgery. 2002;51:S146-54.

12. Thome C, Zevgaridis D, Leheta O, Bazner H, Pöckler-Schöniger C, Wöhrle J, et al. Outcome after less-invasive decompression of lumbar spinal stenosis: a randomized comparison of unilateral laminotomy, bilateral laminotomy, and laminectomy. J Neurosurg Spine. 2015;3:129-41.

\section{Publisher's Note}

Springer Nature remains neutral with regard to jurisdictional claims in published maps and institutional affiliations.

\section{Submit your manuscript to a SpringerOpen ${ }^{\circ}$ journal and benefit from:}

- Convenient online submission

- Rigorous peer review

- Open access: articles freely available online

- High visibility within the field

- Retaining the copyright to your article

Submit your next manuscript at $\boldsymbol{\nabla}$ springeropen.com 